\title{
Molecular-Confinement of Polysulfide within Mesoscale Electrodes for Practical Application of Lithium Sulfur Batteries
}

Junzheng Chen, Dangxin Wu, Eric Walter, Mark Engelhard, Priyanka Bhattacharya, Huilin Pan, Yuyan Shao, Fei Gao, Jie Xiao, Jun Liu*

Joint Center for Energy Storage Research, Pacific Northwest National Laboratory, Richland, WA 99354, USA

\begin{abstract}
Nitrogen-doped porous carbon (NPC) and multi-wall carbon nanotube (MWCNT) have been frequently studied to immobilize sulfur in lithium-sulfur (Li-S) batteries. However, neither NPC nor MWCNT itself can effectively confine the soluble polysufides if cathode thickness e.g. sulfur loading is increased. In this work, NPC was combined with MWCNT to construct an integrated host structure to immobilize sulfur at a relevant scale. The function of doped nitrogen atoms was revisited and found to effectively attract sulfur radicals generated during the electrochemical process. The addition of MWCNT facilitated the uniform coating of sulfur nanocomposites to a practically usable thickness and homogenized the distribution of sulfur particles in the pristine electrodes, while NPC provided sufficient pore volume to trap dissolved species. More importantly, the wetting issue, the critical challenge for thick sulfur cathodes, is also mitigated after the adoption of MWCNT, leading to a high areal capacity of ca. 2.5 $\mathrm{mAh} / \mathrm{cm}^{2}$ with capacity retention of $81.6 \%$ over 100 cycles.
\end{abstract}

* Corresponding authors.

E-Mail: Jun.liu@pnnl.gov (J. Liu)

Tel: +1 (509) 375-4443 


\section{Introduction}

Lithium sulfur batteries have become one of the most promising energy storage technologies, potentially meeting the needs for vehicle electrification and stationary applications, because of its lower costs, environmental friendly property and much higher energy density (cathode theoretical energy: $3500 \mathrm{Wh} / \mathrm{kg}$, or $2800 \mathrm{Wh} / \mathrm{L}$ ) than that of state-of-art Li-ion batteries. However, many challenges exist for the broad deployment of Li/S system. The main challenge is the low Columbic efficiency and fast capacity decay due to the shuttle reactions caused by the soluble intermediate polysulfide [1]. The insulating nature of sulfur requires the addition of a large amount of carbon additive to fully use the active materials [2], which will sacrifice the energy density. In addition, the degradation of original cathode structure due to the loss of sulfur [3] and the continuous impedance increase of lithium metal anode [4] largely limit the lifespan of Li-S cells.

While the Li-S system has to be systematically investigated as a whole, the construction of an effective scaffold to host sulfur in the cathode needs to be firstly addressed. The classical approach by employing ordered mesoporous carbon to confine sulfur can be found in early work from L. Nazar's group [5]. Other forms of carbon materials such as porous carbon [6-8], graphene [9-12], carbon nanotubes or nanofibers [13-15], conductive polymers [16-22] and porous metal oxides [23,24] are also reported. In addition to the surface chemistry [25], the surface area and pore volume are identified to be the most two important attributes in confining sulfur particles. The former directly determines the real current density and thus the polarization of the cell, while the latter provides buffer volume to slow down the diffusion of dissolved sulfur species out of the matrix [26]. It needs to be pointed out that most of the sulfur mass loading in

previous work was limited around $1 \mathrm{mg} \mathrm{cm}^{-2}$ or less, making it difficult to gauge the 
effectiveness of various carbon morphologies. In addition, new challenges such as electrolyte penetration and decreased conductivity rise up in the sulfur electrodes with increased thickness or sulfur contents, which are less addressed in literature.

Herein, we report the synergistic effects of integrated N-doped porous carbon (NPC) and MWCNT to host the sulfur materials. The functioning mechanism of N-doping was revisited in terms of its capability to capture intermediate sulfur radicals. Previous work also reported heteroatom doping to modulate the electron density of carbon substrate $[27,28]$. Multiwallcarbon nanotube (MWCNT) was incorporated in the porous C/S composite to facilitate the construction of thick sulfur electrode (3.6 $\mathrm{mg} / \mathrm{cm}^{2}$ of mass loading), while improving the electrolyte infiltration and electron transport throughout the thick electrodes.

\section{Experimental}

\subsection{Material synthesis and characterization}

Multi-walled CNTs (MWCNT, 10-20nm in diameter, Cheaptubes inc.) were firstly refluxed in 6 $\mathrm{M} \mathrm{HNO}_{3}$ solution for $12 \mathrm{~h}$ to remove impurities and to make them more dispersible in water. NPC-MWCNT was prepared by pyrolyzing disodium magnesium EDTA hydrate (SigmaAldrich) and MWCNT mixture [29]. Typically, disodium magnesium EDTA hydrate was dissolved in the in ethanol: water $(1: 1, \mathrm{v} / \mathrm{v})$ solution, and then pretreated MWCNT was added and sonicated for $15 \mathrm{~min}$. The result solution was transferred to a rotary evaporator and heated at $80^{\circ} \mathrm{C}$ to remove the solvent. The obtained powder was pyrolyzed under $\mathrm{N}_{2}$ protection at $700^{\circ} \mathrm{C}$ for $2 \mathrm{~h}$, followed by washing the products with diluted hydrochloric acid and distilled water till neutral. To investigate the difference, disodium magnesium EDTA hydrate was directly pyrolyzed to obtain NPC carbon. Sulfur was loading by a wet-dry method reported previously[30, 
31]. $\mathrm{CS}_{2}$ solution containing $10 \mathrm{wt} \%$ sulfur was added into the prepared carbon, followed by sonication for $15 \mathrm{~min}$ to form homogeneous slurry. After the evaporation of $\mathrm{CS}_{2}$ in room temperature, the obtained powder was sealed in an autoclave and heated at $155^{\circ} \mathrm{C}$ for $12 \mathrm{~h}$ followed by $300^{\circ} \mathrm{C}$ for $2 \mathrm{~h}$.

Morphology observations were performed with a dual FIB scanning electron microscope (SEM) (FEI Helios). Thermogravimetric analyses (TGA) were conducted on a Netzsch STA 449C thermal analysis system (Netzsch, Germany) under $\mathrm{N}_{2}$ with a temperature rate of $10^{\circ} \mathrm{C}$. All composites contain around $80 \%$ sulfur and 20\% carbon host from their TGA curves in Figure S4b. The nitrogen absorption and desorption isotherms were obtained at $77.3 \mathrm{~K}$ with a QUANTACHROME AUTOSORB 6-B gas sorption system Surface area was determined from

the isotherms using 5 points Brunauer-Emmett-Teller (BET) method. Barrett-Joyner-Halenda (BJH) method was used for the porosity and pore size analyzes based on the adsorption branches. BJH method is a procedure for calculating pore size distribution from experimental isotherm using the Kelvin model of pore filling. T method was used for micro pore analyzes. X-Ray diffraction (XRD) patterns were obtained using a Rigaku Miniflex II X-ray diffractometer with $\mathrm{Cu} \mathrm{K} \alpha$ radiation at $\lambda=1.54 \AA$. XPS measurements were performed with a Physical Electronics Quantera Scanning X-ray Microprobe. Electron paramagnetic resonance (EPR) spectra were obtained with a Bruker Instruments model EMX EPR spectrometer using filter samples placed in quartz tubes.

\subsection{Electrochemical tests}

The cathode was prepared by mixing 80 wt \% composite powder, 10 wt \% Super P (Timcal Graphite \& Carbon), and 10 wt \% polyvinylidene fluoride (PVDF) together in N-methyl-2- 
pyrrolidinone (NMP) to form a slurry, then coated onto the $\mathrm{Al}$ foil collector and dried at $60^{\circ} \mathrm{C}$ under vacuum overnight. The electrode was punched to $0.9 \mathrm{~mm}^{2}$ and the sulfur loading in the electrode is $\sim 2.3 \mathrm{mg} / \mathrm{cm}^{2}$. The electrolyte was $1 \mathrm{M}$ LiTFSI dissolved in a mixture of 1,3dioxolane (DOL) and 1,2-dimethoxyethane (DME) (1:1 v/v), and the separator was Celgard 3400. Half cells with lithium fossil as anode using CR2325 coin-type cell (Canadian National Research Council) were assembled in an argon-filled glovebox, and the discharge/charge cycles were tested galvanostatically using a LANHE Battery tester at room temperature in a voltage cutoff protocol between 3 and $1 \mathrm{~V}$. The specific capacity (SC) was based on the sulfur mass in the C/S composites, and the areal capacity (AC) was calculated by the equation $\mathrm{AC}=\mathrm{SC} \times$ sulfur loading ratio. Electrochemical impedance spectrum (EIS) was measured using the CHI660D electrochemical workstation (CHI660D, CHI Corp.). The AC amplitude was $\pm 5 \mathrm{mV}$, and the applied frequency range was from $100 \mathrm{kHz}$ to $1 \mathrm{~Hz}$.

\subsection{Computational details.}

First-principles calculations were performed based on density functional theory (DFT) as implemented in the VASP code [32] to study the interactions between NPC and $\mathrm{S}_{3}$ - radical. The projector augmented wave method [33,34] and the Perdew-Burke-Ernzerh of exchangecorrelation functions within the generalized gradient approximation (GGA) were used [35]. The plane-wave energy cutoff was set at $400 \mathrm{eV}$ and $\Gamma$-point sampling was used to make sure the total energies were converged to $10^{-4} \mathrm{eV}$. In addition, the dipole correction was applied in the calculations to account for any possible polarity and charger transfer [36].

The interactions between the doped carbon segment and $\mathrm{S}_{3}^{--}$radicals are quantified by calculating the adsorption energies $E_{a}$ as defined by 
$E_{a}=E_{t}-\left(E_{g}+E_{r}\right)$,

where $E_{t}, E_{g}$, and $E_{r}$ are the total energies of the adsorbed systems, the doped graphene segment, and $_{\mathrm{S}_{3} \text {-radical. }}$

\section{Results and discussion}

NPC was synthesized by a facile pyrolysis process of the disodium magnesium EDTA hydrate under nitrogen atmosphere. Differential thermal analysis (DTA) shows four peaks at $170^{\circ} \mathrm{C}$, $\sim 400^{\circ} \mathrm{C}, 450^{\circ} \mathrm{C}$ and $800^{\circ} \mathrm{C}$, which can be assigned to dehydration, decomposition I, II and crystallization (Figure S1a), respectively. Nitrogen content at different pyrolysis temperatures was determined by elemental analysis, which indicates a decrease from 12.1, to 5.6 and only $1.3 \%$ as temperature increases from 600 to $800^{\circ} \mathrm{C}$ (Table 1). $700^{\circ} \mathrm{C}$-derived carbon was used for this work as it contains a balanced surface area and nitrogen content [29]. XRD pattern displays (002) peak at $22.5^{\circ}$ and a small (010) diffraction peak at $42^{\circ}$ (Figure 1a), implying a low graphitization level of carbon pyrolysis in $700^{\circ} \mathrm{C}$. After mixing and heating on the as-prepared carbons and sulfur, it is found that sulfur changes from Fddd orthorhombic phase to monoclinic phase if $700^{\circ} \mathrm{C}$ (or lower)-derived carbon is used. For sulfur in the composite comprised of carbon prepared at $800^{\circ} \mathrm{C}$ or above, sulfur still remains in the orthorhombic (Fddd) phase in the composite. This may be related to the nitrogen content in the carbon, which increases at lower temperatures and monoclinic sulfur was reported to exist in N-containing host [37]. It seems that nitrogen atom influences the phase transition of sulfur during the heating process of S/C composites, suggesting a strong interaction between nitrogen atoms and original sulfur particles. While the mechanism of forming monoclinic sulfur in the presence of concentrated $\mathrm{N}$ atoms on carbon is worthy of further investigation, it has been reported that monoclinic sulfur displayed 
improved cycling stability than its orthorhombic phase, which is a good news for Li-S batteries. XPS results in Figure $\mathbf{1 b}$ and Table S1 show that pyrrolic and pyridinic nitrogen atoms are dominant in the N-doped carbon.

Previous reports on N-doping effects of carbon for sulfur focus on the interactions of nitrogen and oxygen-containing functional groups on the carbon substrate [38]. Considering the empty

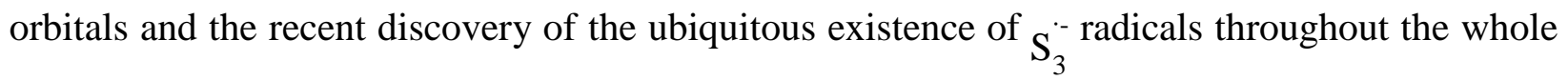
electrochemical processes [39], the interactions between $\mathrm{N}$ atoms and radicals are worth further

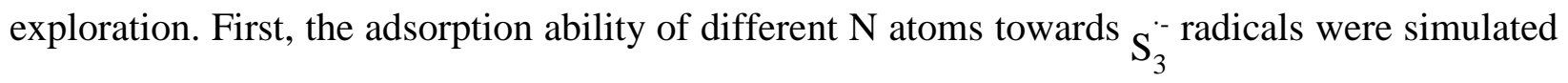
and optimized in Figure 2a, with detailed modeling process in Figure S3 and Table S3. All the optimized configurations have negative adsorption energy, indicating that they are thermodynamically stable. Energetically, $\mathrm{S}_{3}^{-}$radicals prefer to form bond with both pyrrolic- and pyridinic-N atoms from the top of the doped graphene surface, since they had relatively lower adsorption energy of $-2.09 \mathrm{eV}$ and $-2.00 \mathrm{eV}$, respectively. With these effective $\mathrm{N}$-doping sites, the radical intermediate would be stabilized in the carbon shell rather than diffuse away to attack the anode side. The interfacial stability of high sulfur loading electrode is thus improved, which will be discussed later in detail.

Figure 2b shows the EPR results of discharged NPC-S and MWCNT-S composites peeled off from the electrode, which confirms the above theoretical simulation results,. A large signal from carbon free radicals $(g=2.0023)$ were observed in discharged NPC-S and MWCNT-S composites. The two additional shoulder peaks in both cases could also be assigned to the presence of $\mathrm{S}_{3}^{-}$free radicals as reported in the literature $\left(g_{y}=2.0355, g_{z}=2.0526, g_{x}\right.$ was overlapped by carbon signals) [40-42]. The relatively high intensity of the shoulder peaks for 
NPC-S composite in inert left of Figure $2 \mathbf{b}$ imply that more $\mathrm{S}_{3}^{-}$radicals are present in the $\mathrm{N}$ doped carbon hosts, indicating the preferred adsorption of sulfur radical inside the NPC carbon matrix.

Figure 3 compares the morphologies of different carbon hosts before and after loading sulfur. The NPC show coral-like morphology and a rich micropore surface with many large pores in the range of several nanometers (Figure 3a-b). With 80\% sulfur content, the morphology of NPC-S (referred as NPC-80S) in Figure 3c shows a serious agglomeration and most of the surface is covered by sulfur, which implies a low utilization rate of sulfur and barriers for electrolyte to flood in. To address these issues, MWCNTs were integrated into NPC-80S during synthesis. The final ratio of NPC and MWCNT in the carbon mixture is around 1:1. Raman spectrum (Figure S1b) revealed an improved intensity of G-band than that of D-band in the composite, suggesting a higher ratio of graphitic carbon which may improve the electric conductivity. In addition, the XRD spectrum in Figure S4a shows a distinct mixture of amorphous carbon (002) reflex at 22.3 degree broad peak from NPC and sharp peak at 26.2 degree from MWCNT. Besides that, no new phase observed and sulfur kept at Fddd orthorhombic in MWCNT-80S composite, indicating nitrogen promotes the recrystallization of sulfur into monoclinic phase. Raw MWCNT network has a BET surface area of $167.3 \mathrm{~m} 2 / \mathrm{g}$ and the tube surface is well purified from the SEM in Figure 3d. The coverage of sulfur on the MWCNT mitigates the aggregation issue of sulfur imbedded within pure NPC and increases the number of reactive regions through the whole electrode, comparing to the serious agglomeration of sulfur in the large gaps from MWCNT network (Figure 3g). MWCNTs are uniformly integrated into NPC (Figure 3e-f), while maintaining the high surface area and porosity of the whole host structure. After the addition of MWCNT, the surface area of NPC-MWCNT is still high $\left(1418.17 \mathrm{~m}^{2} / \mathrm{g}\right)$ but keeps to $24.95 \mathrm{~m}^{2} / \mathrm{g}$ 
after $80 \%$ sulfur impregnation(Table S2). Figure S1c-d show the $\mathrm{N}_{2}$ adsorption-desorption isotherm and $\mathrm{BJH}$ pore distribution of carbon before and after sulfur loading. The NPC containing carbon all present type I adsorption-desorption isotherms, indicating microporous carbons contribute the most pore volume. In contract, MWCNT reveals obvious sharp peaks at high relative pressure $\left(p / p_{0}>0.9\right)$, which concludes more amount of mesopores at 2-50 nm or even large pores from network structure. After the sulfur loading, NPC carbon still has some micropore volume left, which would be favor for polysulfide reservoirs. The average diameter of MWCNT is 20-40 nm and it has been reported that 2D structure of MWCNT may promote the electrolyte infiltration from the surface to the bulk, which is a challenge in thick sulfur electrodes [31, 43]. The mixture of NPC and MWCNT can expand the reaction zone between sulfur and the electrolyte, and favor the dispersing uniformity of sulfur in the composite. After loading $80 \%$ sulfur into the NPC/MWCNT matrix, sulfur particles demonstrate homogeneous distribution in the carbon host (Figure $3 \mathbf{h}$ ).

The electrochemical performances of NPC-80S, MWCNT-80S and NPC-MWCNT-80S composites are compared in Figure 4. Each parallel test uses electrodes with sulfur loading at ca. $2 \mathrm{mg} / \mathrm{cm}^{2}$. Both areal specific capacity and specific capacity are plotted in Figure 4a. NPC-80S had a very poor initial utilization rate of sulfur, as reflected by the low reversible capacity. This is related to the insufficient electrolyte wetting in the thick sulfur cathode and the poor conductivity of amorphous carbon. A long-term resting of freshly assembled cell does not make obvious improvement, while vacuum cannot be applied to the cell due to the high volatility of ether-based electrolyte. However, the columbic efficiency of NPC-80S is high at $96 \%$ with decent capacity retention of $83 \%$ over 100 cycles. The large surface area and high pore volume alleviate the fast loss of polysulfide, while the intermediate $\mathrm{S}_{3}$ - radicals, dissociated from long- 
chain $\mathrm{S}_{8}{ }^{2-}$ and $\mathrm{S}_{6}{ }^{2-}$ anions [41] are further immobilized through their interactions with $\mathrm{N}$ atoms as discussed before. On the other hand, MWCNT-80S has a high initial specific capacity at 2.3 $\mathrm{mAh} / \mathrm{cm}^{2}$. However, serious shuttle reactions happen which leads to a low Columbic efficiency of only 60\%. The reversible capacity of MWCNT-80S (Figure 4a) decay very quickly with only 50\% capacity retention after 100 cycles. Although MWCNT also has high surface area (187.65 $\mathrm{cm}^{2} / \mathrm{g}$ ), the lacking of porous structures and absence of $\mathrm{N}$ atoms in MWCNT cannot effectively buffer the fast diffusion of dissolved sulfur species towards the anode side. In contrast, when NPC and MWCNT are combined as the host structure, a high Columbic efficiency (> 90\% without $\mathrm{LiNO}_{3}$ additive) has been obtained. The initial capacity also reaches $2.5 \mathrm{mAh} / \mathrm{cm}^{2}$ with stable cycling of $81.6 \%$ retention over 100 cycles. Figure 4c further compares the chargedischarge curves of NPC-MWCNT-80S cathodes after different cycling. The upper plateau at 2.3V corresponds to the conversion of solid elemental sulfur $\left(\mathrm{S}_{8}\right)$ to liquid higher-order lithium polysulfide ( $\mathrm{Li}_{2} \mathrm{~S}_{8}, \mathrm{Li}_{2} \mathrm{~S}_{6}$ ), followed by a liquid to semi-solid $\mathrm{Li}_{2} \mathrm{~S}_{4}$ formation in the slope region. The length of this $2.3 \mathrm{~V}$ plateau does not display significant shrinkage after the first cycle, indicating the effectiveness of molecular-level confinement of soluble polysulfides by doped nitrogen atoms. Otherwise, porous carbon itself still shows quick fading on this upper plateau [30]. The lower plateau at $2.1 \mathrm{~V}$ revealed the semi-solid phase $\mathrm{Li}_{2} \mathrm{~S}_{4}$ further reduction to solid phase low-order $\mathrm{Li}_{2} \mathrm{~S}_{2}$, even $\mathrm{Li}_{2} \mathrm{~S}$. The polarization potential between charge-discharge curves in NPC-MWCNT-80S was significantly reduced to $206 \mathrm{mV}$ comparing to that in NC-80S and MWCNT-80S electrode, which was $220 \mathrm{mV}$ and $340 \mathrm{mV}$, respectively (Figure 4d). The cross section morphology of thick NPC-MWCNT-80S electrodes before and after 100 cycles was shown in Figure 4e-f. The thickness of pressed electrode was about $80 \mu \mathrm{m}$. The EDX results showed that sulfur and carbon was homogeneously mixed. After 100 cycles, the re-deposited 
sulfur particles become smaller due to the electrochemical redistribution process [3]. The electrode thickness, however, has been barely changed, indicating the robustness of the integrated NPC-MWCNT scaffold.

\section{Conclusion}

$\mathrm{N}$-doping effects on carbon substrates have been revisited in this work in terms of its interactions with sulfur radicals generated during the electrochemical reactions in Li-S cells. Theoretical simulation indicates that pyrrolic- and pyridinic- $\mathrm{N}$ atoms doped on the carbon effectively adsorb

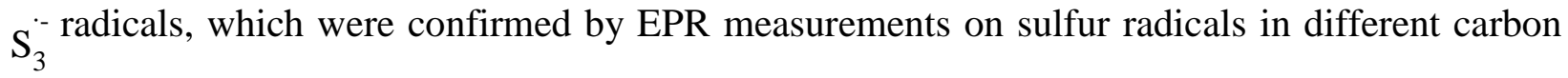
hosts. To better utilize NPC-S composite cathode for practical application, MWCNT is incorporated into thick sulfur electrode to improve the conductivity of the whole electrode as well as facilitate the electrolyte penetration process within the electrode. The distribution of sulfur particles is also modulated after the addition of MWCNT in the electrode, leading to a higher utilization rate and Columbic efficiency. The synergistic effects from NPC and MWCNT lead to a very stable cycling based on a high areal specific capacity of $2.5 \mathrm{mAh} / \mathrm{cm}^{2}$. This work provides clues on the fundamental mechanism of $\mathrm{N}$-doping and the strategies to practically develop nano-structured materials for battery applications.

\section{Acknowledgment}

This work was supported by the Joint Center for Energy Storage Research (JCESR), an Energy Innovation Hub funded by the U.S. Department of Energy (DOE), Office of Science, Basic Energy Sciences (BES). The EPR, XPS, and SEM analyses were performed in the Environmental Molecular Sciences Laboratory (EMSL), a national scientific user facility 
sponsored by the U.S. Department of Energy's Office of Biological and Environmental Research and located at Pacific Northwest National Laboratory (PNNL).

\section{References}

[1] Y. V. Mikhaylik, J. R. Akridge, J. Electrochem. Soc. 151 (2004) A1969-A1976.

[2] X. Li, Y. Cao, W. Qi, L. V. Saraf, J. Xiao, Z. Nie, J. Mietek, J.-G. Zhang, B. Schwenzer, J. Liu, J. Mater. Chem. 21 (2011) 16603-16610.

[3] J. M. Zheng, M. Gu, C. M. Wang, P. J. Zuo, P. K. Koech, J. G. Zhang, J. Liu, J. Xiao, J. Electrochem. Soc. 160 (2013) A1992-A1996.

[4] D. Lv, Y. Shao, T. Lozano, W. D. Bennett, G. L. Graff, B. Polzin, J. Zhang, M. H. Engelhard, N. T. Saenz, W. A. Henderson, P. Bhattacharya, J. Liu, J. Xiao, Adv. Energ. Mater. 10.1002/aenm.201400993(2014).

[5] X. Ji, K. T. Lee, L. F. Nazar, Nat. Mater. 8 (2009) 500-506.

[6] W. Weng, V. G. Pol, K. Amine, Adv. Mater. 25 (2013) 1608-1615.

[7] N. Moreno, A. Caballero, L. Hernan, J. Morales, Carbon 70 (2014) 241-248.

[8] C. D. Liang, N. J. Dudney, J. Y. Howe, Chem. Mater. 21 (2009) 4724-4730.

[9] G. M. Zhou, S. F. Pei, L. Li, D. W. Wang, S. G. Wang, K. Huang, L. C. Yin, F. Li, H. M. Cheng, Adv. Mater. 26 (2014) 625-631.

[10] J. P. Rong, M. Y. Ge, X. Fang, C. W. Zhou, Nano Lett. 14 (2014) 473-479.

[11] M. K. Song, Y. G. Zhang, E. J. Cairns, Nano Lett. 13 (2013) 5891-5899.

[12] R. Chen, T. Zhao, J. Lu, F. Wu, L. Li, J. Chen, G. Tan, Y. Ye, K. Amine, Nano Lett. 13 (2013) 4642-4649.

[13] G. Zheng, Y. Yang, J. J. Cha, S. S. Hong, Y. Cui, Nano Lett. 11 (2011) 44624467. 
[14] L. N. Wang, Y. Zhao, M. L. Thomas, H. R. Byon, Adv. Funct. Mater. 24 (2014) $2248-2252$.

[15] J. Xie, J. Yang, X. Y. Zhou, Y. L. Zou, J. J. Tang, S. C. Wang, F. Chen, J. Power Sources 253 (2014) 55-63.

[16] F. Wu, J. Z. Chen, L. Li, T. Zhao, R. J. Chen, J. Phys. Chem. C 115 (2011) 24411-24417.

[17] F. Wu, J. Z. Chen, L. Li, T. Zhao, Z. Liu, R. J. Chen, Chemsuschem 6 (2013) $1438-1444$.

[18] F. Wu, S. X. Wu, R. J. Chen, J. Z. Chen, S. Chen, Electrochem. Solid State Lett. 13 (2010) A29-A31.

[19] F. Wu, J. Chen, R. Chen, S. Wu, L. Li, S. Chen, T. Zhao, J. Phys. Chem. C 115 (2011) 6057-6063.

[20] W. J. Chung, J. J. Griebel, E. T. Kim, H. Yoon, A. G. Simmonds, H. J. Ji, P. T. Dirlam, R. S. Glass, J. J. Wie, N. A. Nguyen, B. W. Guralnick, J. Park, A. Somogyi, P. Theato, M. E. Mackay, Y. E. Sung, K. Char, J. Pyun, Nat. Chem. 5 (2013) 518-524.

[21] Y. Yang, G. H. Yu, J. J. Cha, H. Wu, M. Vosgueritchian, Y. Yao, Z. A. Bao, Y. Cui, ACS Nano 5 (2011) 9187-9193.

[22] W. Y. Li, Q. F. Zhang, G. Y. Zheng, Z. W. Seh, H. B. Yao, Y. Cui, Nano Lett. 13 (2013) 5534-5540.

[23] K. T. Lee, R. Black, T. Yim, X. L. Ji, L. F. Nazar, Adv. Energ. Mater. 2 (2012) $1490-1496$.

[24] J. Zheng, J. Tian, D. Wu, M. Gu, W. Xu, C. Wang, F. Gao, M. H. Engelhard, J.-G. Zhang, J. Liu, J. Xiao, Nano Lett. 14 (2014) 2345-2352. 
[25] L. Ji, M. Rao, H. Zheng, L. Zhang, Y. Li, W. Duan, J. Guo, E. J. Cairns, Y. Zhang, J. Am. Chem. Soc. 133 (2011) 18522-18525.

[26] J. M. Zheng, D. P. Lv, M. Gu, C. M. Wang, J. G. Zhang, J. Liu, J. Xiao, J. Electrochem. Soc. 160 (2013) A2288-A2292.

[27] Z. Wang, X. Niu, J. Xiao, C. Wang, J. Liu, F. Gao, RSC Adv. 3 (2013) 1677516780.

[28] W. Zhou, X. Xiao, M. Cai, L. Yang, Nano Lett. 14 (2014) 5250-5256.

[29] B. Xu, H. Duan, M. Chu, G. Cao, Y. Yang, J. Mater. Chem. A 1 (2013) 4565.

[30] J. Zheng, M. Gu, M. J. Wagner, K. A. Hays, X. Li, P. Zuo, C. Wang, J. G. Zhang, J. Liu, J. Xiao, J. Electrochem. Soc. 160 (2013) A1624-A1628.

[31] J. Z. Chen, F. Wu, R. J. Chen, L. Li, S. Chen, New Carbon Mater. 28 (2013) 428434.

[32] G. Kresse and J. Hafner, Phys. Rev. B 47 (1993) 558-561.

[33] P.E. Blöchl,Phys. Rev. B 50 (1994) 17953-17979.

[34] G. Kresse and J. Joubert, Phys. Rev. B 59 (1999) 1758-1775.

[35] J.P. Perdew, K. Burke, and M. Ernzerhof, Phys. Rev. Lett. 77 (1996) 3865-3868.

[36] J. Neugebauer and M. Scheffler, Phys. Rev. B 46 (1992) 16067-16080.

[37] W. D. Zhou, Y. C. Yu, H. Chen, F. J. DiSalvo, H. D. Abruna, J. Am. Chem. Soc. 135 (2013) 16736-16743.

[38] J. X. Song, T. Xu, M. L. Gordin, P. Y. Zhu, D. P. Lv, Y. B. Jiang, Y. S. Chen, Y. H. Duan, D. H. Wang, Adv. Funct. Mater. 24 (2014) 1243-1250.

[39] M. A. Lowe, J. Gao, H. D. Abruña, RSC Adv. 4 (2014) 18347. 
[40] M. Vijayakumar, N. Govind, E. Walter, S. D. Burton, A. Shukla, A. Devaraj, J.

Xiao, J. Liu, C. Wang, A. Karim, S. Thevuthasan, Phys. Chem. Chem. Phys. 16 (2014) 1092310932.

[41] C. Barchasz, F. Molton, C. Duboc, J. C. Lepretre, S. Patoux, F. Alloin, Anal. Chem. 84 (2012) 3973-3980.

[42] J. Goslar, S. Lijewski, S. K. Hoffmann, A. Jankowska, S. Kowalak, J. Chem. Phys. 130 (2009) 204504.

[43] F. Wu, A. Magasinski, G. Yushin, J. Mater. Chem. A 2 (2014) 6064-6070. 
Table 1 Percentage composition of the elements in the various composites

\section{Elemental Analysis}

\begin{tabular}{ccccc} 
Sample & \multicolumn{4}{c}{ (by weight) } \\
& $\mathbf{C}$ & $\mathbf{H}$ & $\mathbf{N}$ & $\mathbf{O}$ \\
\hline NPC-600 & 75.44 & 2.56 & 12.08 & 9.92 \\
NPC-700 & 86.0 & 1.55 & 5.84 & 6.61 \\
NPC-800 & 92.75 & 0.56 & 1.21 & 5.48 \\
NPC-900 & 95.43 & 0.27 & 0.76 & 3.54 \\
\hline
\end{tabular}



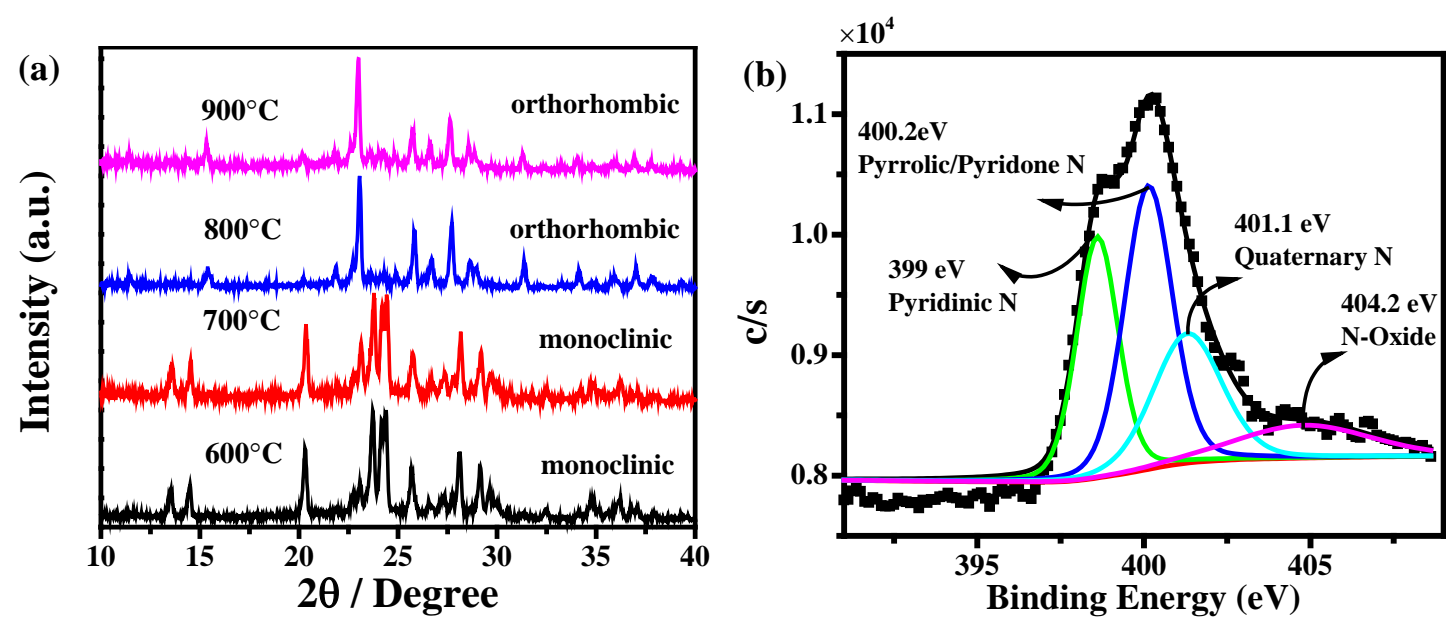

Figure 1(a) XRD spectrum of different pyrolysis temperature NPC-80S composite (b) XPS spectrum of N 1s characteristic peaks in NPC composite. 
(a)

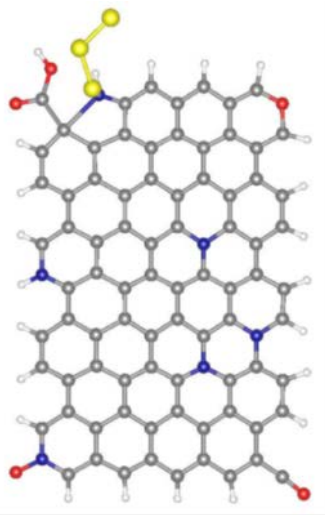

Binding Energy $=-2.09 \mathrm{eV}$

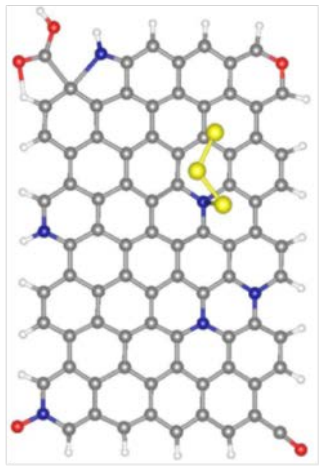

$-2.00 \mathrm{eV}$

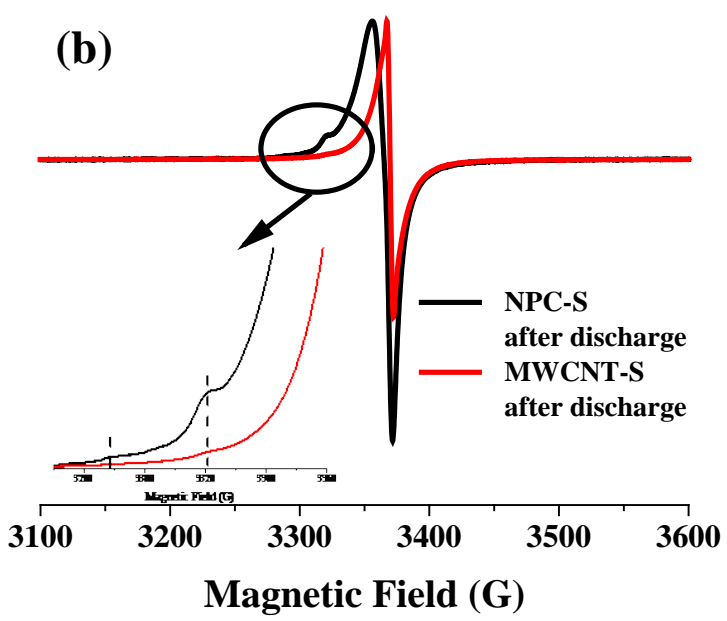

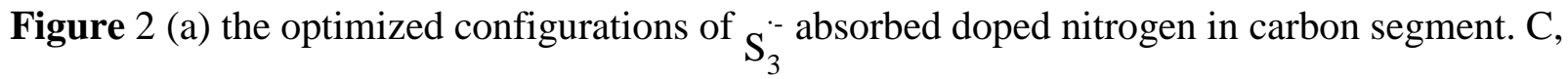
$\mathrm{H}, \mathrm{O}, \mathrm{N}, \mathrm{S}$ atoms are represented by grey, white, red, blue and yellow spheres, respectively. (b) EPR spectrum of NPC/S and MWCNT/S composite after fully discharged, showing the presence

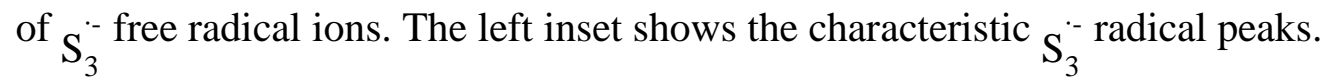



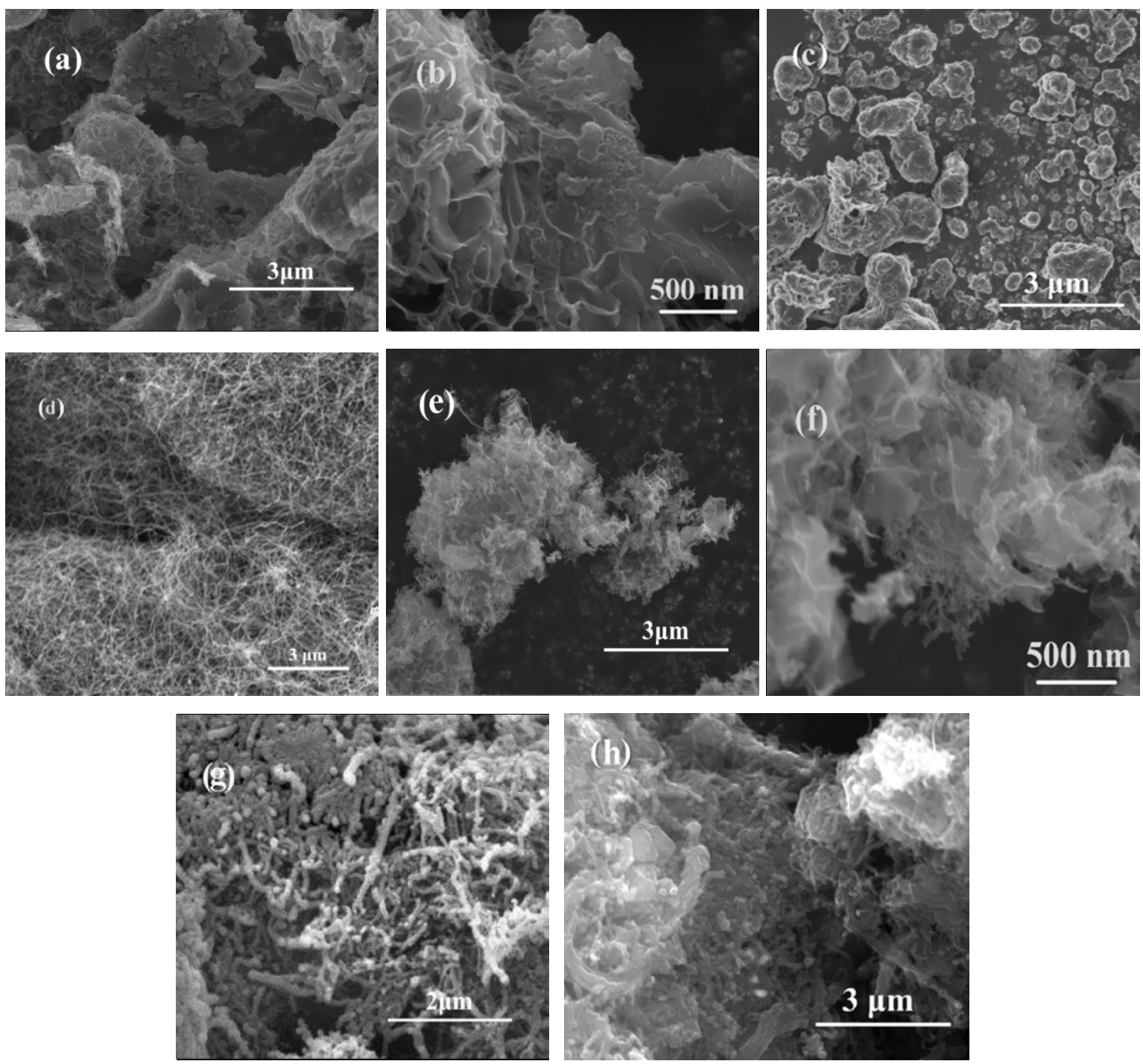

Figure 3 SEM image of (a, b) NPC composite (c)NPC-80S composite (d)MWCNT (e, f)NPCMWCNT composite (g)MWNCT-80S (h)NPC-MWNCT-80S composite 

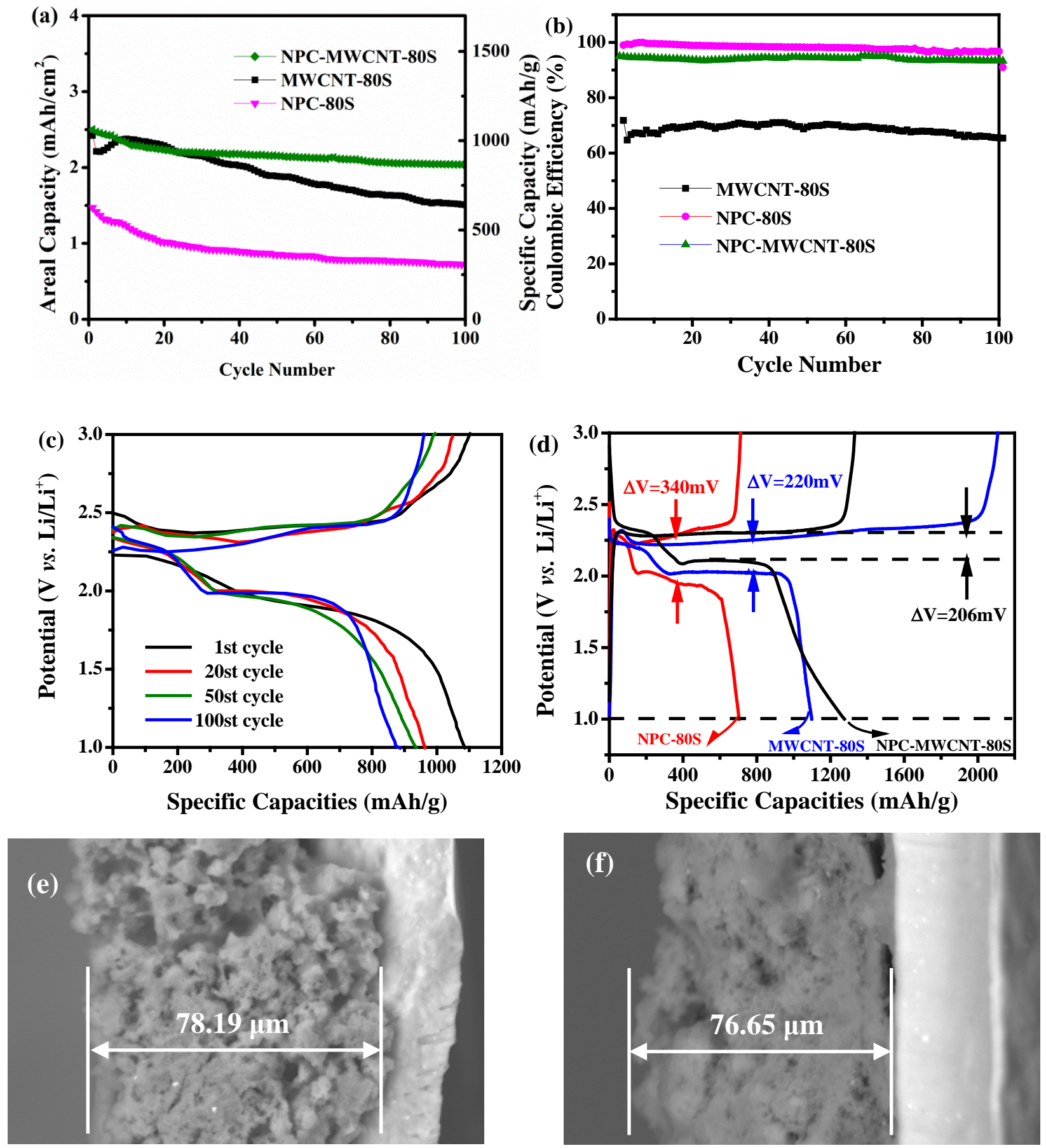

Figure 4 (a) Cycling performance and (b) corresponding Coulombic efficiency of sulfur containing composite under $3.6 \mathrm{~mA} / \mathrm{cm}^{2}$ at a voltage cutoff between $1.0-3.0 \mathrm{~V}$ (c) cycle voltage profile for NPC-80S composite under $3.6 \mathrm{~mA} / \mathrm{cm}^{2}$ (d) initial Charge/discharge voltage profile evolution for different composite during cycling at $0.36 \mathrm{~mA} / \mathrm{cm}^{2}$ (e) cross section SEM images of pristine electrode (f) and the electrode after 100 charge/discharge cycles 